Archives de sciences sociales des religions

176 | octobre-décembre 2016

Bulletin Bibliographique

\title{
Rosanne Kanhai (eds.), Bindi. The Multifaceted Lives of Indo-Caribbean Women
}

Kingston, University of the West Indies Press, 2011, 256 p.

Mathieu Claveyrolas

\section{OpenEdition}

Journals

Édition électronique

URL : http://journals.openedition.org/assr/28251

DOI : 10.4000/assr.28251

ISSN : $1777-5825$

Éditeur

Éditions de l'EHESS

Édition imprimée

Date de publication : 31 décembre 2016

Pagination : 323

ISSN : 0335-5985

Référence électronique

Mathieu Claveyrolas, « Rosanne Kanhai (eds.), Bindi. The Multifaceted Lives of Indo-Caribbean Women », Archives de sciences sociales des religions [En ligne], 176 | octobre-décembre 2016, mis en ligne le 18 juillet 2017, consulté le 24 septembre 2020. URL : http://journals.openedition.org/assr/28251 ; DOI : https://doi.org/10.4000/assr.28251

Ce document a été généré automatiquement le 24 septembre 2020.

(C) Archives de sciences sociales des religions 


\title{
Rosanne Kanhai (eds.), Bindi. The Multifaceted Lives of Indo- Caribbean Women
}

Kingston, University of the West Indies Press, 2011, 256 p.

\author{
Mathieu Claveyrolas
}

\section{RÉFÉRENCE}

Rosanne Kanhai (eds.), Bindi. The Multifaceted Lives of Indo-Caribbean Women, Kingston, University of the West Indies Press, 2011, 256 p.

1 L'ouvrage édité par Rosanne Kanhai doit être resitué dans l'histoire des publications sur les cultures nées de l'engagisme indien. Longtemps négligées, ces dernières suscitent depuis une vingtaine d'années un indéniable intérêt - en partie nourri par l'accès de ces communautés à la visibilité sur la scène publique voire, à Trinidad ou à Maurice notamment, à une certaine hégémonie politique et culturelle. L'angle d'attaque thématique et disciplinaire ici privilégié - les femmes et les gender studies est également nouveau quoique ponctuant déjà une première décennie de recherches, prolongeant notamment le premier ouvrage collectif de Rosanne Kanhai : Matikor: The Politics of Identity for Indo-Caribbean Women, publié en 1999.

2 L'ouvrage comprend, outre l'introduction de l'éditrice, neuf chapitres répartis en trois parties. L'ensemble, quoiqu'hétéroclite, voire inégal, reste souvent très intéressant.

3 Dans la première partie ("Religion in a global context»), Sherry-Ann Singh explore en détail le rôle des femmes hindoues dans la persistance, la transmission et l'adaptation du Ramayana à Trinidad depuis l'engagisme alors que Halima Sa'adia Kassim se penche sur les schémas et stratégies maritales de la communauté indo-musulmane à Trinidad, et sur leur évolution au gré de la mobilité sociale et des influences de la globalisation.

4 La seconde partie ("Constructing Self») compte trois courts chapitres qui resserrent la méthodologie sur un nombre restreint d'individus. Les voix des femmes indo- 
trinidadiennes qu'on y entend suffisent à la rendre intéressante. Shaheeda Hosein analyse le rôle et le statut des femmes au début $d u x^{e}$ siècle dans la sphère domestique en milieu rural ; elle fonde ses matériaux sur quelques interlocutrices âgées (dont des proches). Valérie Youssef se livre à un exercice un peu artificiel, interrogeant brièvement ( 30 minutes minimum», p. 125) trois de ses étudiantes indotrinidadiennes en linguistique (une hindoue, une musulmane, une pentecôtiste) sur leurs perceptions identitaires (nationales, religieuses, ethniques). Enfin, Gabrielle Jamela Hosein rend compte avec finesse d'une observation participante originale : son inscription et sa victoire au concours de beauté réservé aux femmes indotrinidadiennes.

5 La troisième et dernière partie ("Survival and Creativity ») regroupe deux chapitres d'analyses du champ des productions littéraires des femmes indo-trinidadiennes (par Anita Baksh et Paula Morgan), un chapitre d'analyse de la vie et de l'œuvre de l'artiste guyanaise Bernadette Persaud (par Brenda Gopeesingh) et un autre ethnographiquement intéressant, mais peu problématisé et plutôt mal intégré à cette partie - sur les femmes thérapeutes (et sages-femmes) traditionnelles à Trinidad (écrit par le seul homme à participer : Kumar Mahabir).

On remarque d'emblée que le titre et le sous-titre de l'ouvrage ne vont pas sans discussion. Le lecteur pourrait d'abord s'attendre à davantage de représentativité et de diversité derrière l'analyse promise des femmes « indo-caribéennes »; en fait, au moins 7 des 9 chapitres du volume se concentrent sur les femmes indo-trinidadiennes, et les deux autres se contentent d'intégrer deux femmes indo-guyanaises. La discussion aurait probablement gagné à comparer, même ponctuellement, avec le reste de la Caraïbe, et notamment les contextes où les communautés indo-caribéennes ne sont pas majoritaires.

7 Bien qu'évocateur, le titre suscite également la discussion. Le premier ouvrage collectif de Kanhai était intitulé Matikor du nom d'une danse réservée aux femmes qui, lors d'une des soirées de mariage, mimaient l'acte sexuel. Le bindi, lui, est une marque portée par les femmes sur le front, au milieu et en surplomb des sourcils. Rosanne Kanhai choisit le bindi comme symbole de cette féminité dont elle souhaite ici analyser les diverses dimensions chez les Indo-Caribéennes. Le bindi, pourtant, n'est qu'une version du tilak (ou tika) qui, dans l'hindouisme, signifie l'alliance. À ce titre, il orne aussi bien le front des hommes qui ont rendu leur dévotion (symbolisant l'alliance ainsi scellée avec la divinité) que celui des femmes mariées (signalant alors l'alliance avec leur époux - la poudre rouge est remplacée par de la cendre lors du veuvage). Rosanne Kanhai évoque en introduction le "pouvoir du bindi d'échapper aux frontières culturelles et de véritablement appartenir à toutes les femmes » (p. 2). Sans négliger la portée esthétique du bindi, et le succès de ses dérivés autocollants récents au-delà des femmes hindoues mariées, on peut se demander si l'auteur ne va pas un peu vite en besogne lorsqu'elle le détache de toute valeur hindoue (et sociale ou, dirait-elle, " patriarcale ») pour en faire le symbole trop univoque d'une féminité à la fois libérée et transcendant les frontières ethniques.

8 Sans doute rejoint-on là une certaine tendance aux raccourcis qui émaillent le volume. L'« effet-Trinidad", d'abord, est souvent durci - prêtant au nouveau contexte des engagés des dimensions qui préexistaient pourtant en Inde. Ainsi quand Sherry-Ann Singh explique la consommation d'alcool et de viande qui accompagne certains rituels par le «brouillage entre le religieux et le séculier, entre le sacré et le profane, entre 
l'orthodoxie prescrite et la culture populaire» (p.32) qui aurait caractérisé les premiers stades de l'hindouisme trinidadien. On aurait aimé voir précisé que le cas n'est pas exceptionnel en Inde-même, pour peu que l'on se penche sur certaines castes et traditions populaires - celles, justement, qui ont été très représentées dans l'engagisme. De même, Shaheeda Hosein parle-t-elle de la «nature ignominieuse de la veuve ", une " idéologie héritée de l'Inde ", un "stigma » (p. 115) dont les femmes indotrinidadiennes auraient su se libérer en se remariant ou, à défaut, en s'installant en concubinage après la mort de leur mari. Là aussi, il aurait été bon de préciser que l'interdit du remariage des veuves touche particulièrement en Inde les hautes castes, et que les basses castes engagées y sont souvent étrangères.

On regrette ensuite parfois que le choix militant des auteurs d'analyser et comprendre tout phénomène par le seul biais des gender studies tende à durcir l'analyse. Ainsi Kumar Mahabir conclut-il un peu artificiellement son papier sur les femmes thérapeutes en proposant sans convaincre qu'on puisse lire dans leur activité un «mouvement de protestation finement déguisé contre les médecins hommes professionnels» (p. 177). Rosanne Kanhai elle-même introduit cette volonté de privilégier l'analyse féministe sur la spécificité culturelle en prétendant trop rapidement que la « déesse-terre [sic] Kali représente l'éternelle féminité au-delà des cultures » (p. 2).

10 Cela dit, le propos de l'ouvrage, et la problématique gender studies, ouvrent effectivement des chantiers passionnants. Quand l'anthropologue haïtien Michel-Rolph Trouillot soulignait combien la logique coloniale visait à "faire taire le passé » (Silencing the past, 1995), il mettait le doigt sur une constante de l'histoire des sociétés à fondement esclavagiste. Comment des milliers d'hommes, esclaves, mais aussi leurs descendants, majoritaires et indispensables à un processus de production qui résume toute la société, ont-ils pu à ce point disparaître de l'histoire et de ses récits? La question reste en partie valable pour l'engagisme, légèrement déplacée par le fait que les engagés indiens débarqués à l'abolition de l'esclavage se sont retrouvés tout à la fois « à la périphérie » et " tout en bas » (p. 145) d'un ordre social créole construit sans eux dans le face à face entre Blancs et Noirs. Les femmes, surtout, apparaissent comme muettes et invisibles par excellence parmi ces communautés silencieuses. On assigne d'ailleurs aux femmes les mêmes qualificatifs qu'aux esclaves : "passives, obéissantes " (p. 199), mais aussi « résilientes». Les femmes engagées sont les sous-prolétaires du système capitaliste de la plantation, tout au bas de la hiérarchie ethnique coloniale, et sous le coup d'une logique patriarcale propre à leur culture. On pourra retenir trois chapitres, traitant en particulier de chacune de ces dimensions.

11 Concernant les femmes dans le système capitaliste issu de la plantation d'abord, Shaheeda Hosein porte le regard sur la sphère domestique - la plus silencieuse. Ce chapitre ambitieux intitulé « Unlikely Matriarchs » nous convainc du dynamisme de ces femmes, acteurs invisibles, mais cruciaux, non seulement au quotidien de la famille et de la communauté, mais à la re-production de ses valeurs. L'auteur nous fait partager le quotidien de ces femmes : les exemples du lavage des vêtements durant les règles (à l'abri des regards), ou des empreintes de mains dont ces femmes décoraient les murs des maisons comme signatures de leur "domaine ", dessinent un tableau nuancé des rôles et statuts (dont celui, crucial, de belle-mère) de la femme indo-trinidadienne.

Gabrielle Jamela Hosein propose, elle, un chapitre réflexif joliment intitulé « No Pure Place of Resistance » retraçant sa participation au Mastana Bahar Indian Cultural Pageant 2000. Dans ce « contre-concours » défiant les standards dominants (Euro-Américains et 
Afro-Trinidadiens) de beauté à Trinidad (p. 148), l'auteur traque efficacement l'incessante négociation de la « féminité indienne » qui se joue sur scène et en coulisses. Elle défie également les normes féministes habituelles, gagnant le titre de «militant maharani » dans les journaux locaux, mais recevant un accueil plus mitigé de militantes critiquant sa participation à un concours de beauté (p. 151).

13 Retenons enfin le premier chapitre de l'ouvrage dans lequel Sherry-Ann Singh étudie la contribution des femmes dans l'évolution de la tradition du Ramayana « à la fois comme miroir et métaphore de l'expérience hindoue à Trinidad" (p.45). Là encore, la contribution des femmes à la transmission de la tradition ne se fait pas sans une réappropriation, sans ces « détours » qui font penser aux modes de résistance créoles. Les thématiques sur lesquelles reposent la récitation, l'inclusion de dimensions proprement trinidadiennes, les interprétations et les performances oratoires (notamment des femmes) défient l'ordre et la morale patriarcaux du récit, transformant Sita - épouse vertueuse et soumise - en symbole de l'indépendance féminine.

14 Ce volume pose donc d'excellentes questions et offre plusieurs pistes de réponses ou d'enquêtes à venir. Reste une interrogation face à l'évolution du statut et du rôle des femmes d'origines indiennes dans les diasporas engagées. À l'heure des affirmations identitaires tous azimuts cherchant en Inde une «authenticité » perdue maintenant que l'histoire de l'engagisme semble passée, les élites qui promeuvent et administrent ce changement de paradigme sont très majoritairement masculines: comment l'histoire des femmes indo-trinidadiennes, de leur résistance et de leur empowerment, va-t-elle s'insérer dans ce nouveau projet communautaire? 